\title{
Crystal structure and functional implications of the tandem-type universal stress protein UspE from Escherichia coli
}

Yongbin $\mathrm{Xu}^{1,2^{*}}$, Jianyun Guo ${ }^{1}$, Xiaoling $\mathrm{Jin}^{1}$, Jin-Sik Kim${ }^{3}$, Ying Ji ${ }^{1}$, Shengdi Fan ${ }^{1}$, Nam-Chul Ha ${ }^{3}$ and Chun-Shan Quan ${ }^{1}$

\begin{abstract}
Background: The universal stress proteins (USP) family member UspE is a tandem-type USP that consists of two Usp domains. The UspE expression levels of the Escherichia coli (E. coli) become elevated in response to oxidative stress and DNA damaging agents, including exposure to mitomycin C, cadmium, and hydrogen peroxide. It has been shown that UspA family members are survival factors during cellular growth arrest. The structures and functions of the UspA family members control the growth of $E$. coli in animal hosts. While several UspA family members have known structures, the structure of E. coli UspE remains to be elucidated.

Results: To understand the biochemical function of UspE, we have determined the crystal structure of E. coli UspE at $3.2 \AA$ resolution. The asymmetric unit contains two protomers related by a non-crystallographic symmetry, and each protomer contains two tandem Usp domains. The crystal structure shows that UspE is folded into a fan-shaped structure similar to that of the tandem-type Usp protein PMl1202 from Proteus mirabilis, and it has a hydrophobic cavity that binds its ligand. Structural analysis revealed that $E$. coli UspE has two metal ion binding sites, and isothermal titration calorimetry suggested the presence of two $\mathrm{Cd}^{2+}$ binding sites with a $\mathrm{K}_{d}$ value of 38.3-242.7 $\mu \mathrm{M}$. Structural analysis suggested that E. coli UspE has two $\mathrm{Cd}^{2+}$ binding sites (Site I: His117, His 119; Site Il: His193, His244).

Conclusion: The results show that the UspE structure has a hydrophobic pocket. This pocket is strongly bound to an unidentified ligand. Combined with a previous study, the ligand is probably related to an intermediate in lipid A biosynthesis. Subsequently, sequence analysis found that UspE has an ATP binding motif (Gly ${ }^{269}-\mathrm{X}_{2}-\mathrm{Gly}^{272}-\mathrm{X}_{9}-\mathrm{Gly}^{282}$-Asn) in its C-terminal domain, which was confirmed by in vitro ATPase activity monitored using Kinase-Glo ${ }^{\otimes}$ Luminescent Kinase Assay. However, the residues constituting this motif were disordered in the crystal structure, reflecting their intrinsic flexibility. ITC experiments revealed that the UspE probably has two $\mathrm{Cd}^{2+}$ binding sites. The His117, His 119, His 193, and His244 residues within the $\beta$-barrel domain are necessary for $\mathrm{Cd}^{2+}$ binding to UspE protein. As mentioned above, USPs are associated with several functions, such as cadmium binding, ATPase function, and involvement in lipid A biosynthesis by some unknown way.
\end{abstract}

Keywords: UspE, UspA superfamily, Tandem-type USP

\footnotetext{
* Correspondence: yongbinxu@dlnu.edu.cn

'Department of Bioengineering, College of Life Science, Dalian Nationalities University, Dalian 116600Liaoning, China

${ }^{2}$ Laboratory of Biomedical Material Engineering, Dalian Institute of Chemical

Physics, Chinese Academy of Sciences, Dalian 116023Liaoning, China

Full list of author information is available at the end of the article
} 


\section{Background}

The universal stress proteins (USP) superfamily is a group of conserved proteins that play an important role in $E$. coli. USPs' expression levels become elevated in response to a bewildering variety of stress conditions, such as heat shock, nutrient starvation, the presence of oxidants, DNA-damaging agents (including exposure to mitomycin $\mathrm{C}$, cadmium, and hydrogen peroxide), as well as others, that may arrest cell growth. Proteins in the UspA family constitute a natural biological defense mechanism $[1,2]$. Despite considerable research on the behavior of UspA family members, the biological and biochemical roles of these proteins remain largely uncharacterized. Very few details were available to help decipher their roles in the aforementioned cellular processes [1]. A better understanding of the molecular mechanisms of E. coli's UspA proteins is important for establishing effective therapeutic strategies. In particular, establishing the three-dimensional structural model of the UspE protein can provide hints to explore the function(s) of the UspA family.

E. coli has six small UspA superfamily genes: uspA, $-C$ $-D,-E,-F$, and $-G$. To date, these proteins have been extensively investigated. Previous studies have shown that UspA family members show immaculate similarity. They encode either a small USP protein (approximately 14 to $15 \mathrm{kDa}$ ) that consists of two USP domains in tandem or a larger version (approximately $30 \mathrm{kDa}$ ) that consists of two peptides attached as a single functional protein [3, 4]. UspA, UspC, and UspD belong to class I; UspF and UspG belong to class II; two Usp domains of UspE belong to class II and IV based on the sequence and structural analysis $[3,5,6]$. Previous works have found that while Usp family members have partially overlapping functions, the functions of class I, II, and IV Usps are distinct [7]. UspA proteins differ in their responses to protect cells from oxidative stress and DNA damage agents; UspA, UspC, UspD, and UspE are induced by exposure to mitomycin $\mathrm{C}$, cadmium, and hydrogen peroxide. However, class II proteins, UspG and UspF, were associated with iron scavenging in the cell [4]. As mentioned before, UspE is a tandem-type USP. When UspE proteins are split apart and treated separately, the UspE2 domain is more closely related to UspF and UspG. This is clearly visible in both the clustering analysis and the reconstructed cladogram. In contrast, UspE1 groups are more closely related to class I UspA proteins (UspACD) [1].

This paper includes structural and functional studies on UspE from E. coli. Specifically, it presents the threedimensional X-ray crystal structure of the recombinantly produced UspE from E. coli at $3.2 \AA$ resolution. Additionally, through the use of structural biochemical analyses, the UspE mechanisms were determined. In terms of its overall structure, UspE was found to be similar to the tandem-type Usp protein PMl1202 from Proteus mirabilis, which has a hydrophobic cavity that binds an unidentified ligand. It was also observed that UspE has an ATP binding motif (Gly ${ }^{269}$-Thr-Val-Gly ${ }^{272}-\mathrm{X}_{9}-\mathrm{Gly}^{282}$ Asn) in its C-terminal domain. The ATPase activity was then measured to determine if UspE had ATPase activity and to characterize UspE activity. Because previous research found that UspE is critical for $\mathrm{Cd}^{2+}$ defense, we characterized the role of UspE as part of the $\mathrm{Cd}^{2+}$ binding process by ITC and structural analysis and found that UspE has two $\mathrm{Cd}^{2+}$ binding sites in its tandem USP domain. These observations suggest that UspE performs several distinct functions, such as ATP hydrolysis and cadmium defense. Although the molecular function of this protein remains unknown, our three-dimensional structures of UspE offer valuable clues to understand its potential biochemical mechanisms.

\section{Methods}

Structure determination, refinement and protein data bank accession number of UspE

We have previously reported the crystallization and preliminary X-ray analysis of E. coli UspE [8]. Data collection and refinement statistics are summarized in Table 1. The structure of the E. coli UspE protein was determined by molecular replacement (MR) using the program CCP4 package [9]. We used the coordinates of the structures of $P$. mirabilis PMl1202 (78.5 \% sequence identity; PDB code: 3OLQ) as a reference. To build our protein model, we first removed model bias by rounds of simulated annealing performed with the program PHENIX [10], followed by calculating the differences using Fourier maps. Then, the UspE model was rebuilt in the graphic program COOT [11]. The model was finally refined using the same programs by iterative rounds of energy minimization, Bfactor, and anisotropic refinements. Then, the composite omit and differences were calculated by Fourier maps. UspE coordinates and structure have been deposited in the Protein Data Bank [12] under accession code: 5CB0 (www.rcsb.org/pdb).

\section{Isothermal titration calorimetry}

Isothermal titration calorimetry (ITC) measurements were performed on a Microcal iTC 200 (GE Healthcare) VP-ITC microcalorimeter at $298 \mathrm{~K}$. The protein was dialyzed against $20 \mathrm{mM}$ Hepes (pH 7.0) and $150 \mathrm{mM} \mathrm{NaCl}$. The titration $\mathrm{CdCl}_{2}$ solution was prepared with $20 \mathrm{mM}$ Hepes (pH 7.0) and $150 \mathrm{mM} \mathrm{NaCl}$ by adding $2 \mathrm{mM}$ $\mathrm{CdCl}_{2}$. Both the protein and the titrant $\mathrm{CdCl}_{2}$ solutions were thoroughly degassed in a ThermoVac apparatus (Microcal). The titration reaction was performed by sequential injections of $40 \mu \mathrm{CdCl}_{2}$ solution into the sample cell. The duration of the injection was $120 \mathrm{~s}$. The 
Table 1 Diffraction statistics

\begin{tabular}{|c|c|}
\hline X-ray source & $\begin{array}{l}\text { Beamline 5C, Pohang } \\
\text { Accelerator Laboratory }\end{array}$ \\
\hline PDB code & $5 C B 0$ \\
\hline Wavelength $(\AA)$ & 1.000 \\
\hline Space group & 14,22 \\
\hline Resolution $(\AA)$ & 19.9-3.2 \\
\hline Parameters $(\AA)$ & $a=b=121.1 \AA, c=241.7 \AA, a=\beta=\gamma=90^{\circ}$ \\
\hline Rsym (\%) & $15.3 \%(33.4 \%)$ \\
\hline Completeness (\%) & $92.7(90.2)$ \\
\hline Redundancy & $5.3(3.6)$ \\
\hline Average $1 / \sigma(I)$ & $9.2(3.3)$ \\
\hline R-factor (\%) & 24.31 \\
\hline Rfree (\%) & 30.06 \\
\hline Rmsd for bonds $(\AA)$ & 0.007 \\
\hline Rmsd for angles ( ) & 1.393 \\
\hline \multicolumn{2}{|l|}{ Ramachandran plot (\%) } \\
\hline Favored regions & $93.64 \%$ \\
\hline Allowed regions & $5.12 \%$ \\
\hline Disallowed regions & $1.24 \%$ \\
\hline \multicolumn{2}{|l|}{ Number of atoms } \\
\hline Protein & 4630 \\
\hline Ligand & 34 \\
\hline Average B factor $\left(\AA^{2}\right)$ & 70.54 \\
\hline
\end{tabular}

$+R_{\text {merge }}=\sum_{h k \mid} \Sigma_{i}\left|l_{i}(h k l)-<l(h k l)>\right| \sum_{h k \mid} \Sigma_{i} \mid l_{i}(h k l)$, where $\mid(h k l)$, where I(hkl) is the intensity of reflection $h k l, \Sigma_{h k l}$ is the sum over all reflections and $\Sigma_{1}$ is the sum over $i$ measurements of reflection $h k l . \neq R_{\text {work }}=\Sigma_{h k l}\left|F_{\mathrm{o}}-F_{\mathrm{c}}\right| / \Sigma_{h k l}\left|F_{\mathrm{o}}\right|$ for all data with $F_{\mathrm{o}}>2 \sigma\left(F_{\mathrm{o}}\right)$, excluding data used to calculate $R_{\text {free. }}$. $R_{\text {free }}=\Sigma_{h k l} \mid F_{\mathrm{o}^{-}}$ $F_{\mathrm{c}}\left|/ \Sigma_{h k l}\right| F_{\mathrm{o}} \mid$ for all data with $F_{\mathrm{o}}>2 \sigma\left(F_{\mathrm{o}}\right)$ that were excluded from refinement

syringe was rotated at $600 \mathrm{rev} \mathrm{min}^{-1}$. Triplet measurements were collected in each case.

\section{Kinase-Glo ${ }^{\circledR}$ luminescent kinase assay}

The in vitro ATPase activity of UspE was measured by quantifying the amount of ATP remaining in the solution following a kinase reaction using a Kinase-Glo ${ }^{\circ} \mathrm{Lu}$ minescent Kinase Assay Kit (Promega, Fitchburg, WI, USA). The assay was performed in a 96-well plate in a kinase reaction volume of $50 \mu \mathrm{l}$ containing $10 \mathrm{mM}$ $\mathrm{MgCl}_{2}, 5 \mu \mathrm{M}$ ATP,10 mM HEPES (pH 8.0) and $150 \mathrm{mM}$ $\mathrm{NaCl}$. The reaction was initiated by adding the protein to a final concentration of $0.4 \mathrm{mg} / \mathrm{ml}-3.2 \mathrm{mg} / \mathrm{ml}$. The reaction mixture was kept at $310 \mathrm{~K}$ for $20 \mathrm{~min}$ in a water bath. Reaction mixtures containing no UspE were used as negative controls. The kinase reaction mixture was incubated with $50 \mu \mathrm{l}$ of ATP detection reagent. The plates were then incubated for another $10 \mathrm{~min}$ at $310 \mathrm{~K}$. The Synergy2 Multi-Mode Microplate Reader (BioTek, Winooski, VT, USA) was used to collect the relative light unit (RLU) signal. The luminescent signal was positively correlated with the amount of remaining ATP and inversely correlated with the amount of kinase activity.

\section{Results and discussion Overall structure}

To examine the biochemical mechanisms responsible for UspE function, we determined the crystal structure of the UspE by the molecular replacement method with the synchrotron data set at a resolution of $3.2 \AA$. The final model refined to a R-factor of $0.24\left(R_{\text {free }}=0.30\right)$. The initial solution suggested the presence of two monomers per asymmetric unit, which is consistent with the Matthews' coefficient of $3.1 \AA^{3} \mathrm{Da}^{-1}$ (60.37\% solvent). The tertiary structure of the UspE is very similar to that of the previously described P. mirabilis Usp protein PMI1202 (PDB code: 3OLQ), which was used as a search model in molecular replacement $[13,14]$. UspE exists as a monomer, and the structure reveals a compact and 2-fold symmetric dimer in the crystal. Each monomer consists of two USP domains, and the final model contains two homologous subunits related by a non-crystallographic symmetry (Fig. 1a). The E. coli UspE has a high structural similarity compared with the P. mirabilis USP (PDB code: 4WY2) (Fig. 1b). The crystal structure shows that UspE is folded into a fan-shaped structure similar to that of the tandemtype Usp protein USP from $P$. mirabilis (Fig. 1c). In a Ramachandran plot, $93.82 \%$ of the model residues were found in favored regions, $5.72 \%$ in allowed regions, and $1.06 \%$ in the disallowed regions. Their structures are virtually identical with a root-mean-square deviation (RMSD) value of $0.40 \AA$ for a $244 \mathrm{C} \alpha$ atom. UspE is composed of ten $\beta$-stranded mixed $\beta$-sheet and nine $\alpha$-helices. In the core structure, ten $\beta$-strands form a central parallel $\beta$-sheet (Fig. 1d). Significant density was observed for all residues in the final electron density map except 163-170, $202-215$, and $270-282$. Table 1 provides refinement statistics and structure solution for all structures.

\section{E. coli UspE accommodates an unidentified ligand}

More importantly, we found an unambiguous stick-like electron density in the hydrophobic pocket of E. coli UspE throughout the refinement process. It looks like that the UspE carries the unidentified ligand. The crystal structure of $P$. mirabilis USP suggested that Uridine-5' -diphosphate-3-O-(R-3-hydroxymyristoyl)-

$\mathrm{N}$-acetyl-D-glucosamine was tightly bound to $P$. mirabilis USP. We found that the Uridine- $5^{\prime}$-diphosphate3-O-(R-3-hydroxymyristoyl)-N-acetyl-D-glucosamine binding pocket of $P$. mirabilis USP was very similar to the hydrophobic pocket of E. coli UspE (Fig. 2a) and that the hydrophobic pocket of $E$. coli UspE was appropriate for binding Uridine-5'-diphosphate-3-O-(R-3-hydroxymyristoyl)-N-acetyl-D-glucosamine. In addition, the stick-like electron densities were very similar to a 3- 
a
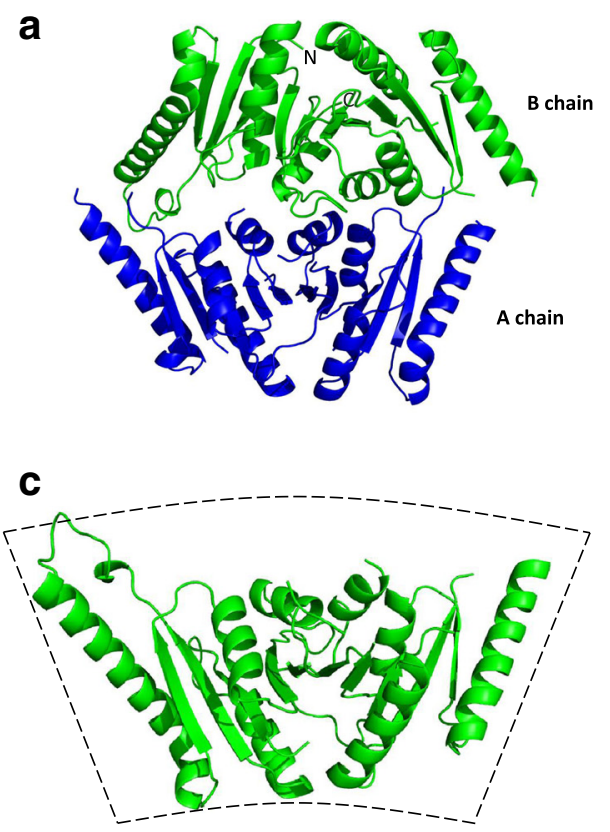

b
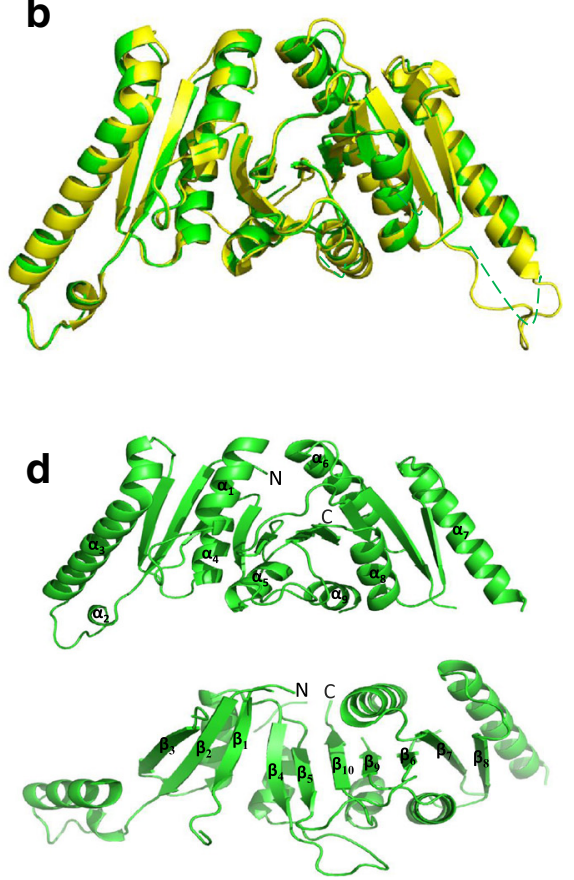

Fig. 1 Overall structure of the UspE. a Structure of EcUspE in the tetragonal crystal form, displayed as ribbons. The asymmetric unit contains two protomers colored blue and green. $\mathbf{b}$ Structural comparison of the cartoon traces of EcUspE and P. mirabilis USP (PDB code: 4WY2). Ec UspE is colored green, and the P. mirabilis USP is colored yellow. The disordered regions are shown with dashed lines. c The monomer structure of EcUspE. $\mathbf{d}$ Secondary structural elements of UspE are numbered

hydroxymyristoyl group of Uridine-5' -diphosphate-3-O-(R3-hydroxymyristoyl)-N-acetyl-D-glucosamine. Thus, this group was placed at density result. Despite the fact that $E c \mathrm{UspE}$ crystals were obtained in the absence of 3hydroxymyristoyl group molecules in both media and buffers, these positions could be successfully refined with no significant residual difference density and with associated B-factors comparable with those of the surrounding atoms. The characteristic hydrophobic environment in the pocket indicates that UspE can bind unidentified ligand with a 3-hydroxymyristoyl group in the cavity. This pocket was surrounded by hydrophobic residues in $\beta 6, \beta 9, \beta 10$, and $\alpha 6$ (Fig. $2 b$ and c). This finding indicates that hydrophobic interactions are involved in the binding of this ligand. A previous study using mass spectrometric and surface analyses showed that the UspE homologue protein

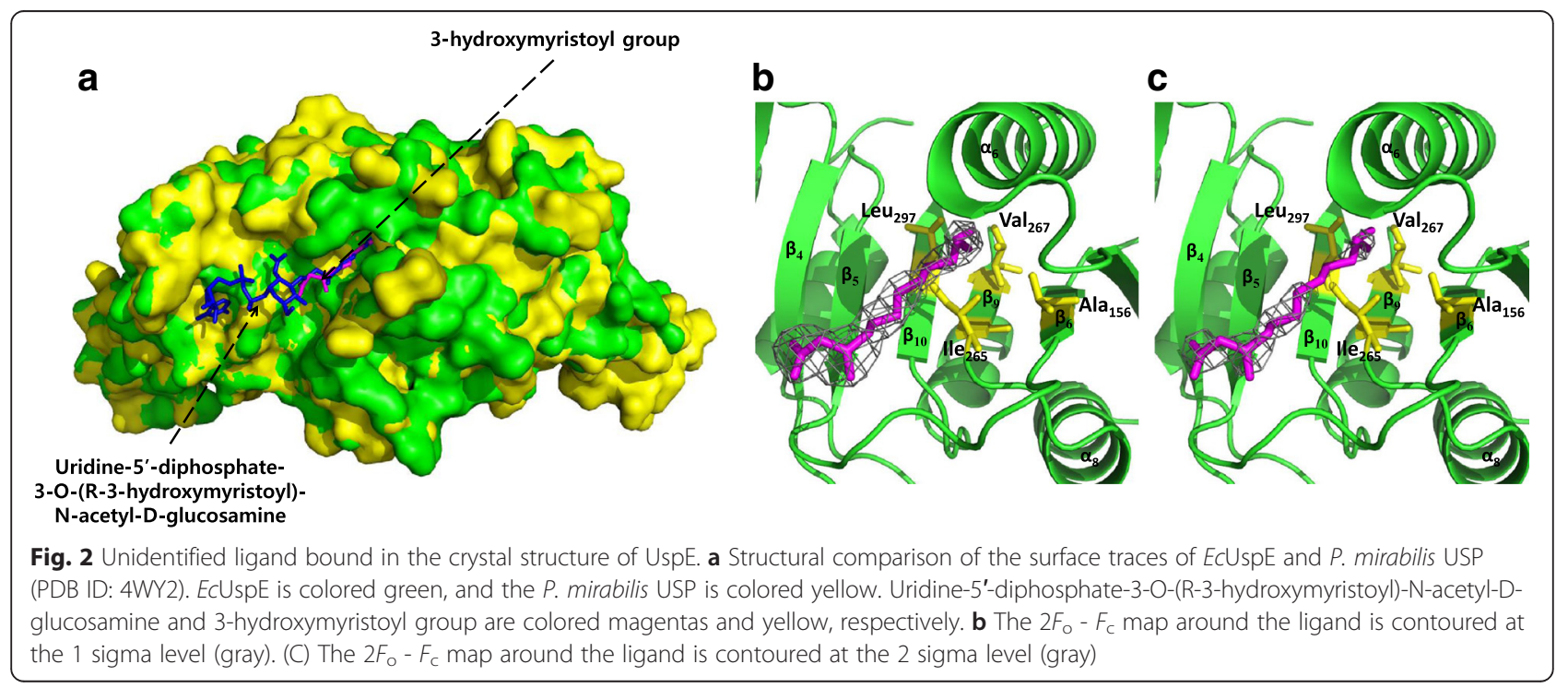


YdaA from Salmonella enterica serovar Typhimurium might bind a large, non-polar ligand in its $\mathrm{N}$-terminal domain; however, YdaA was not bound to any ligand in crystal structure [15]. The ligand bound to $P$. mirabilis USP was tentatively identified as UDP-(3-O-(R3-hydroxymyristoyl))-N-acetylglucosamine. The published crystal structures of the LpxA and LpxC from E. coli contain UDP-(3-O-(R-3-hydroxymyristoyl))-Nacetylglucosamine and its deacetylated product, respectively $[16,17]$. These proteins catalyze the first committed step of lipid A biosynthesis [18]. Combined with these data, the unidentified ligand bound to $E$. coli UspE is probably related to an intermediate in lipid A biosynthesis, similar to UDP-(3-O-(R-3hydroxymyristoyl))- $\mathrm{N}$-acetylglucosamine deacetylases.

\section{E. coli UspE has an ATP binding motif and ATPase activity} Previous studies show that USPs can be divided into groups: those that bind ATP (UspFG-type), those that do not bind ATP (UspAs and UspA-like group), and those that hydrolyze adenine nucleotide substrates. USPs that bind ATP may function as an ATP-dependent signaling intermediate in a pathway that promotes persistent infection. Furthermore, it was suggested that USPs contain a conserved-sequence Gly- $\mathrm{X}_{2}$-Gly- $\mathrm{X}_{9}$-Gly (Ser/ Thr/Asn) motif that is needed for binding ATP [3, 5, 6, $15,19,20]$. However, the ATP binding motif of UspE was disordered in the crystal, due to the intrinsic flexibility of these regions (Fig. 3a). Therefore, it is likely that the bound ATP is disordered or the protein devoid of bound ATP was preferentially crystallized. In this structure, we found the presence of this motif $\left(\mathrm{Gly}^{269}-\mathrm{X}_{2^{-}}\right.$ $\mathrm{Gly}^{272}-\mathrm{X}_{9}-\mathrm{Gly}^{282}$-Asn) in the C-terminal domain of UspE, which is similar to other USPs that bind ATP (Fig. 3b). The in vitro ATPase activity of UspE was determined by measuring the amount of ATP left in solution following a kinase reaction using the Kinase-Glo ${ }^{\circ}$ Luminescent Kinase Assay. As expected, the decline in luminescent signal depended on the increasing concentration of UspE (Fig. 3c). These results indicate that ATPase activity from UspE decreases the remaining ATP levels.

\section{UspE has putative $\mathrm{Cd}^{2+}$ binding sites}

Cadmium, in a variety of chemical forms, is toxic for the proper growth of microbial cells. Previous studies showed that cadmium $(273 \mu \mathrm{M})$ can cause complete but transient inhibition of growth accompanied by the synthesis of cadmium-induced proteins (CDPs) [21]. The $E$. coli increase synthesis of CDPs (e.g., H-NS, UspA, UspC, UspD, UspE), which together make up the cadmium stress stimulon [7]. The UspE can sequester Cd from the cytosol to protect themselves. To analyze the relationship between UspE and cadmium, we investigated the cadmium binding ability of UspE by an ITC experiment.

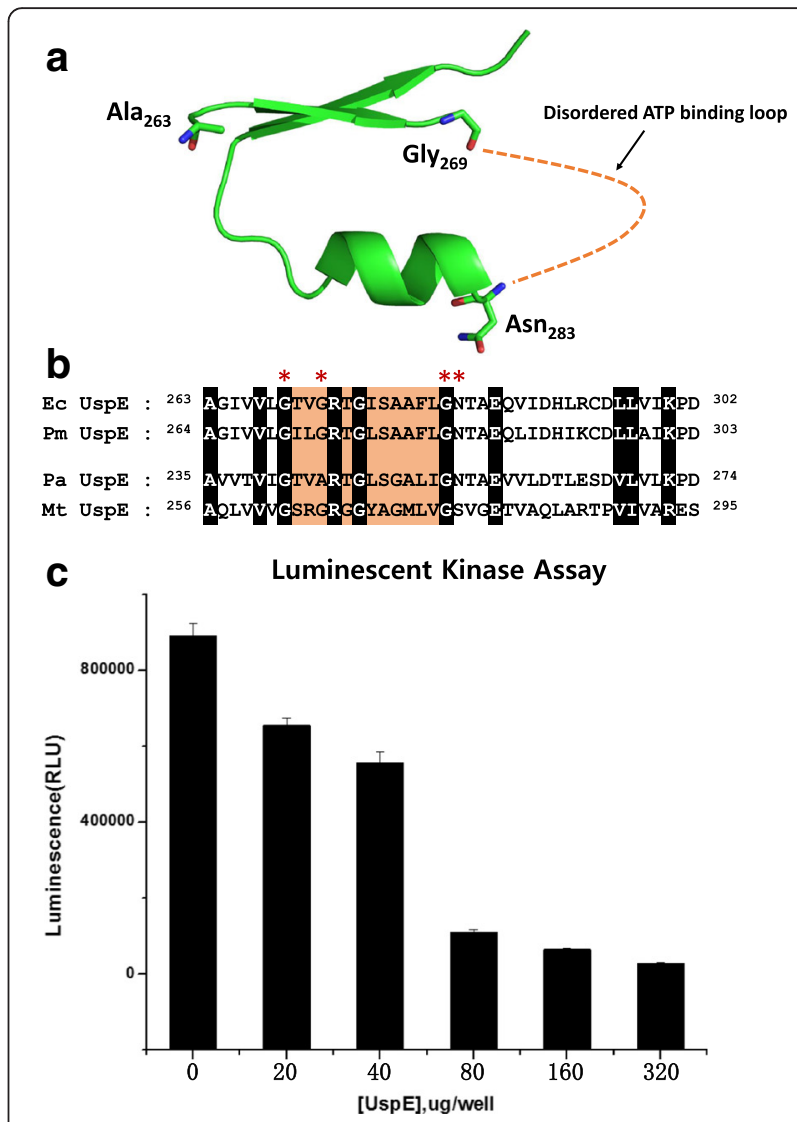

Fig. 3 ATP binding motif and ATPase activity of UspE. a Close-up view of the $\mathbf{b}$ Alignment of the sequences around the ATP binding motif from E. coli UspE (Ec UspE), P. mirabilis (Pm UspE), P. aeruginosa UspE (Pa UspE), and M. tuberculosis (Mt UspE). The conserved residues and ATP binding motif are highlighted. c Kinase activity of UspE. Varying concentrations $(0.4,0.8,1.6,3.2,6.4, \mathrm{mg} / \mathrm{ml})$ of UspE were used in the reaction mixture, and the protein are resuspended in $50 \mu \mathrm{l}$ containing $10 \mathrm{mM} \mathrm{MgCl} 2$ and $5 \mu \mathrm{M}$ ATP. The graph represents the mean of three independent experiments, and the standard deviation is indicated by error bars

The ITC experiment was carried out using cadmium as titrant at $\mathrm{pH}$ 7.0. Initial attempts to fit the data to a double site-binding model were not successful. The isotherm was best fit when a sequential binding model with three binding sites was applied. Through analysis of these ITC data, we observed tight $\mathrm{Cd}^{2+}$ binding to UspE with $n=3$. This suggests a binding stoichiometry of 3 moles of $\mathrm{Cd}^{2+}$ to 1 mole of UspE. The two have moderate binding affinities ( $\mathrm{K}_{\mathrm{d}}$ of 33.7 and $94.3 \mu \mathrm{M}$, respectively), whereas the other one has low affinity $\left(K_{d}\right.$ of $\left.242.7 \mu \mathrm{M}\right)$. The $K_{d}$ value of site 1 and site 2 are high compared with site 3 . At site 1 and site 2, the binding of $\mathrm{Cd}^{2+}$ with UspE is favorable, with an exothermic enthalpy $\left(\Delta \mathrm{H}\right.$ of -18.2 and $-9.384 \mathrm{kcal} \mathrm{mol}^{-}$ 1 , respectively) and negative entropy $(\Delta S=-12.67$ and $-3.25 \mathrm{kcal} \mathrm{mol}^{-1}$, respectively). At site 3 , the binding of $\mathrm{Cd}^{2+}$ with UspE is unfavorable, with an exothermic enthalpy $\left(\Delta \mathrm{H}=-8.131 \mathrm{kcal} \mathrm{mol}^{-1}\right)$ and negative entropy 
Table 2 ITC experiment $\mathrm{Cd}^{2+}$ binding to UspE

\begin{tabular}{lllll}
\hline & $\mathrm{Kd}$ & $\mathrm{H}$ & $\mathrm{T} \mathrm{S}$ & $\mathrm{G}$ \\
& $\mu \mathrm{M}$ & $\mathrm{kcal} / \mathrm{mol}$ & $\mathrm{kcal} / \mathrm{mol}$ & $\mathrm{kcal} / \mathrm{mol}$ \\
\hline Site 1 & 94.3 & -18.2 & -12.67 & -5.5 \\
Site 2 & 33.7 & -9.383 & -3.25 & -6.1 \\
Site 3 & 242.7 & -8.131 & -3.19 & -5.0 \\
\hline
\end{tabular}

$\left(\Delta \mathrm{S}=-3.19 \mathrm{kcal} \mathrm{mol}^{-1}\right)$. The detailed thermodynamic parameters are listed in Table 2 and Fig. 4a. Evidence has been reported that to obtain lethal effects in an exponentially growing culture, $600 \mu \mathrm{M} \mathrm{CdCl}_{2}$ is required. In the lag phase before growth commenced, $3 \mu \mathrm{M}$
$\mathrm{CdCl}_{2}$ inhibited cell proliferation and $10 \mu \mathrm{M}$ was lethal $[13,14]$. To regulate the concentration of $\mathrm{Cd}^{2+}, \mathrm{E}$. coli might induce CDPs such as UspE. These results suggest that apoUspE and $\mathrm{Cd}^{2+}$ have a direct relationship. To understand the relationship between apoUspE and $\mathrm{Cd}^{2+}$, we observed the ability of cadmium to interact with UspE through the ITC experiment. Our structural analysis indicates that $E$. coli has two putative binding sites (Site I: His117, His 119; Site II: His193, His244) (Fig. 4b). Additionally, sequence alignment showed that residues His117, His119, His193, and His244 within the $\beta$-barrel domain are highly conserved among the UspE proteins (Fig. 4c). Recently, a study has been performed to observe site I,

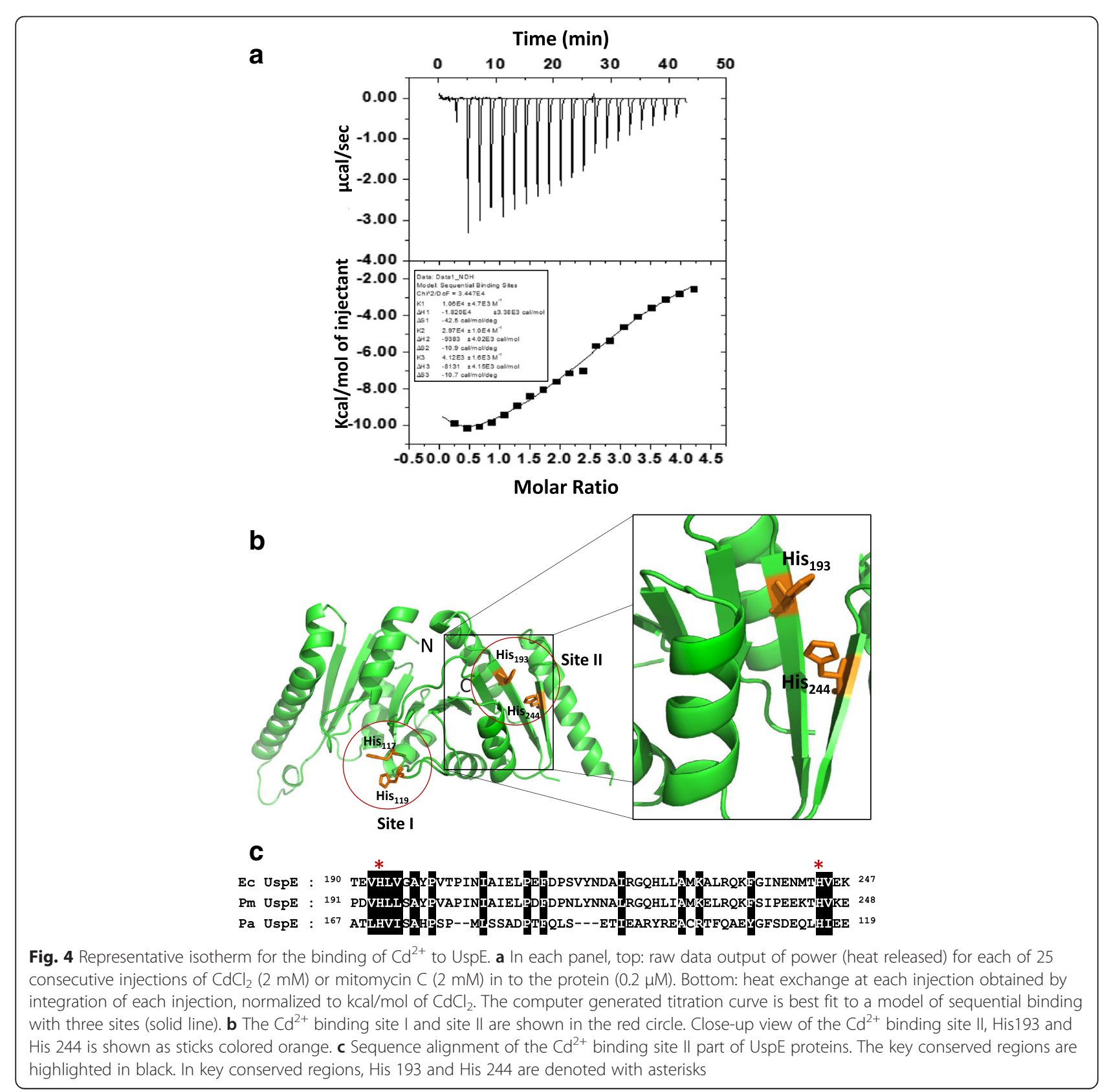


which is known to be crucial for zinc binding in the crystal structure of YdaA from S. enterica serovar Typhimurim (PDB ID: 4R2J) [15]. This provides further support for the original conclusion that cadmium binds at two locations. Our results clearly demonstrate that $E$. coli UspE has two different sets of binding sites and the protein may provide additional confirmation for the cadmium binding to these two sites.

In summary, the crystal structure of UspE from E. coli is representative of a tandem-type USP. The crystal structure of E. coli UspE reveals a hydrophobic pocket that moderately binds an unidentified ligand. Combined with previous studies, we can conclude that UspE is probably related to an intermediate in lipid A biosynthesis. We subsequently found through the sequence analysis that UspE has an ATP binding motif ( Gly $^{269}$. $\mathrm{X}_{2}$-Gly ${ }^{272}-\mathrm{X}_{9}-\mathrm{Gly}^{282}$-Asn) in the C-terminal domain and has ATPase activity, though this did not appear in the crystal structure. We were also able to perform an ITC experiment which revealed that UspE probably has two $\mathrm{Cd}^{2+}$ binding sites and that the His117, His119, His193, and His244 residues within the $\beta$-barrel domain are critical for binding $\mathrm{Cd}^{2+}$. We believe that this information is a significant contribution to understanding the molecular mechanisms of $E$. coli UspE.

\section{Conclusions}

In this study, we have determined the crystal structure of UspE of $E$. coli as a representative of a tandem-type USP. The UspE consists of two tandem USP domains that are highly conserved in this protein family. We found a hydrophobic pocket in the UspE structure, which was strongly bound to unidentified ligand. Combined with a previous study, evidence suggests that the UspE is related to an intermediate in lipid A biosynthesis. We subsequently found that sequence analysis suggests that UspE has an ATP binding motif $\left(\mathrm{Gly}^{269}\right.$ $\mathrm{X}_{2}-\mathrm{Gly}^{272}-\mathrm{X}_{9}$-Gly ${ }^{282}$-Asn) in the C-terminal domain of UspE and has ATPase activity, but this was not confirmed by the crystal structure. We were also able to perform the ITC experiment, which revealed that the UspE probably has two $\mathrm{Cd}^{2+}$ binding sites, comprised of the His117, His 119, His193, and His244 residues within the $\beta$-barrel domain. Both of them are essential for $\mathrm{Cd}^{2+}$ binding to UspE protein. As discussed before, USPs might be associated with several functions, such as cadmium binding, ATPase activity, and an intermediate in lipid A biosynthesis.

\section{Abbreviations}

E. coli: Escherichia coli; ITC: isothermal titration calorimetry; MR: molecular replacement; P. mirabilis: Proteus mirabilis; PDB: protein data bank: RLU: relative light unit; RMSD: root-mean-square deviation; S. enterica serovar Typhimurim: Salmonella enterica serovar Typhimurium; USP: universal stress protein.

\section{Competing interests}

The authors declare that they have no competing interests.

\section{Authors' contributions}

$J G, X J$, and $Y J$ carried out the protein expression, purification, crystallization, and biochemical experiments. YX and JK performed structural modeling and analyzed data. YX, SF, NH, and CQ helped write the manuscript. All authors read and approved the final manuscript.

\section{Acknowledgements}

We gratefully acknowledge the access to the beamline $5 \mathrm{C}$ at Pohang Light Source (PLS) (Pohang, South Korea). This study was supported by the National Natural Science Foundation of China (Grant No. 31200556 to Y. Xu, Grant No.31301447 to Y. Ji, and Grant No. 21172028 to Shengdi Fan), the China Postdoctoral Science Foundation (Grant No. 2013 M540229 to Y. Xu), and the Fundamental Research Funds for the Central Universities (Grant No. DC201502020203 to Y. Xu).

\section{Author details}

'Department of Bioengineering, College of Life Science, Dalian Nationalities University, Dalian 116600Liaoning, China. ${ }^{2}$ Laboratory of Biomedical Material Engineering, Dalian Institute of Chemical Physics, Chinese Academy of Sciences, Dalian 116023Liaoning, China. ${ }^{3}$ Department of Agricultural Biotechnology, College of Agriculture and Life Sciences, Seoul National University, Gwanak-gu, Seoul 151-742, Republic of Korea.

Received: 17 July 2015 Accepted: 1 February 2016

Published online: 11 February 2016

\section{References}

1. Tkaczuk KL, Shumilin IA, Chruszcz M, Evdokimova E, Savchenko A, Minor W. Structural and functional insight into the universal stress protein family. Evol Appl. 2013;6(3):434-49.

2. Gustavsson N, Diez A, Nystrom T. The universal stress protein paralogues of Escherichia coli are co-ordinately regulated and co-operate in the defence against DNA damage. Mol Microbiol. 2002:43(1):107-17.

3. Kvint K, Nachin L, Diez A, Nystrom T. The bacterial universal stress protein: function and regulation. Curr Opin Microbiol. 2003;6(2):140-5.

4. Nachin L, Nannmark U, Nystrom T. Differential roles of the universal stress proteins of Escherichia coli in oxidative stress resistance, adhesion, and motility. J Bacteriol. 2005;187(18):6265-72.

5. Sousa MC, McKay DB. Structure of the universal stress protein of Haemophilus influenzae. Structure. 2001;9(12):1135-41.

6. Zarembinski TI, Hung LW, Mueller-Dieckmann HJ, Kim KK, Yokota H, Kim R, et al. Structure-based assignment of the biochemical function of a hypothetical protein: a test case of structural genomics. Proc Natl Acad Sci U S A. 1998:95(26):15189-93.

7. Siegele DA. Universal stress proteins in Escherichia coli. J Bacteriol. 2005;187(18):6253-4.

8. $X u$ Y, Quan CS, Jin X, Jin X, Zhao J, Li X, et al. Crystallization and preliminary $X$-ray diffraction analysis of UspE from Escherichia coli. Acta Crystallogr F Struct Biol Commun. 2014;70(Pt 12):1640-2.

9. McCoy AJ, Grosse-Kunstleve RW, Adams PD, Winn MD, Storoni LC, Read RJ. Phaser crystallographic software. J Appl Crystallogr. 2007 Aug 1; 40(Pt 4): 658-74.

10. Adams PD, Afonine PV, Bunkoczi G, Chen VB, Davis IW, Echols N, et al PHENIX: a comprehensive Python-based system for macromolecular structure solution. Acta Crystallogr D Biol Crystallogr. 2010;66(Pt 2):213-21.

11. Emsley P, Cowtan K. Coot: model-building tools for molecular graphics. Acta Crystallogr D Biol Crystallogr. 2004;60(Pt 12 Pt 1):2126-32.

12. Berman HM, Westbrook J, Feng Z, Gilliland G, Bhat TN, Weissig H, et al. The protein data bank. Nucleic Acids Res. 2000;28(1):235-42.

13. Lo YC, Lin SC, Shaw JF, Liaw YC. Crystal structure of Escherichia coli thioesterase I/protease I/lysophospholipase L1: consensus sequence blocks constitute the catalytic center of SGNH-hydrolases through a conserved hydrogen bond network. J Mol Biol. 2003:330(3):539-51.

14. Lo YC, Lin SC, Shaw JF, Liaw YC. Substrate specificities of Escherichia coli thioesterase I/protease //lysophospholipase L1 are governed by its switch loop movement. Biochemistry. 2005;44(6):1971-9.

15. Bangera M, Panigrahi R, Sagurthi SR, Savithri HS, Murthy MR. Structural and functional analysis of two universal stress proteins $\mathrm{YdaA}$ and $\mathrm{YnaF}$ from 
Salmonella typhimurium: possible roles in microbial stress tolerance. J Struct Biol. 2015;189(3):238-50.

16. Williams AH, Raetz CR. Structural basis for the acyl chain selectivity and mechanism of UDP-N-acetylglucosamine acyltransferase. Proc Natl Acad Sci U S A. 2007;104(34):13543-50

17. Clayton GM, Klein DJ, Rickert KW, Patel SB, Kornienko M, Zugay-Murphy J, et al. Structure of the bacterial deacetylase LpxC bound to the nucleotide reaction product reveals mechanisms of oxyanion stabilization and proton transfer. J Biol Chem. 2013;288(47):34073-80.

18. Gao N, McLeod SM, Hajec L, Olivier NB, Lahiri SD, Bryan Prince D, et al. Overexpression of Pseudomonas aeruginosa $\mathrm{LpxC}$ with its inhibitors in an acrB-deficient Escherichia coli strain. Protein Expr Purif. 2014;104C:57-64.

19. Weber A, Jung K. Biochemical properties of UspG, a universal stress protein of Escherichia coli. Biochemistry. 2006;45(6):1620-8.

20. Saveanu C, Miron S, Borza T, Craescu CT, Labesse G, Gagyi C, et al. Structural and nucleotide-binding properties of YajQ and YnaF, two Escherichia coli proteins of unknown function. Protein Sci. 2002:11(11):2551-60.

21. Ferianc $P$, Farewell A, Nystrom $T$. The cadmium-stress stimulon of Escherichia coli K-12. Microbiology. 1998;144(Pt 4):1045-50.

\section{Submit your next manuscript to BioMed Central} and we will help you at every step:

- We accept pre-submission inquiries

- Our selector tool helps you to find the most relevant journal

- We provide round the clock customer support

- Convenient online submission

- Thorough peer review

- Inclusion in PubMed and all major indexing services

- Maximum visibility for your research

Submit your manuscript at www.biomedcentral.com/submit 